\title{
The Effect of a Rise in Prices upon the Amount of Small Money used.
}

\author{
By Dr Lester R. Ford.
}

\section{(Read 8th June 1917. Received 14th June 1917.)}

At the close of the preceding meeting of the Society a discussion arose concerning the effect of a uniform rise in prices upon the amount of small money necessary for the transaction of business. It was clear that the total amount of money must be increased, but in the case of small money, which is used only when fractional parts of the larger unit are involved, the effect was less obvious. It is the fact, however, that more small money is required. The present paper attempts an explanation of that fact.

We shall take $£ 1$ as the monetary unit, and consider as small money any coin or other money of lesser value. Let us consider the factors determining the amount of small money involved in an ordinary transaction. A purchase is made for which a payment of $x$, which we will suppose less than $£ l$, is to be made. If the small money in the purchaser's possession amounts to more than $x$, the sum $x$ will be paid therefrom. If, however, his stock of small money is less than $x$, a pound note will be tendered, and small money to the amount of $1-x$ will be given him. In like manner, if $1+x$ or $2+x$ is the amount to be paid, the amount of small money used will be $x$ or $1-x$, according to the purchaser's stock, the additional units not being paid in small money. There will be exceptions to this procedure, but it is doubtless followed in the great majority of cases.

It follows from this that each individual will always have less than $£ 1$ in small money in his possession. With numerous trans. actions each individual's stock varies rapidly between the limits 0 and 1 ; and it can be reasonably assumed that for any large 
number of persons chosen at random, the amounts held are fairly evenly distributed over the interval $0-1$, that those whose stock amounts to between 5 and 6 shillings, for example, are about as numerous as those having between 13 and 14 shillings.*

Now let

$$
y=f(x)
$$

be the frequency curve representing the total number of transactions of the nation in, say, one year, with the sums paid as abscissae; that is, the number of transactions which require a payment of between $x$ and $x+d x$ is $f(x) d x$. The transactions represented by the curve should be only those involving money, payments by cheque, for example, being excluded.

Of the total number of persons making the $f(x) d x$ payments of sums between $x$ and $x+d x$ (we assume $x<1$ for the present), the uniform distribution of the stocks of small money over the interval $0-1$ informs us that $x f(x) d x$ will have less than $x$ of small money, and $(1-x) f(x) d x$ will have more than $x$. Each of the former transactions involves, as we have seen, the payment of $1-x$ in small money; and each of the latter involves the payment of $x$. Hence all the former require the payment of $(1-x) x f^{\prime}(x) d x$, and all the latter require the same sum. Then the total amount of small money required for the payments between $x$ and $x+d x$ is

$$
2 x(1-x) f(x) d x \text {. }
$$

And the total small money required for all payments of less than $f 1$ is

$$
2 \int_{0}^{1} x(1-x) f(x) d x .
$$

The small money required for payments between $£ 1$ and $£ 2$ depends, as we have seen, upon the fractional parts of $£ 1$ involved, and upon the frequency curve between 1 and 2 . It amounts to

$$
2 \int_{0}^{1} x(1-x) f(x+1) d x .
$$

The total small money required for all transactions amounts to

$$
2 \int_{0}^{1} x(1-x)[f(x)+f(x+1)+f(x+2)+\ldots] d x .
$$

* This assumption is discussed at the end of the paper. 
Now suppose that there is a change of conditions. Let $y=\phi(x)$ be the new frequency curve; then the amount of small money used will be

$$
2 \int_{0}^{1} x(1-x)[\phi(x)+\phi(x+1)+\phi(x+2)+\ldots] d x .
$$

Let us suppose that this change is due solely to a uniform change in prices-that all prices are multiplied by $k$. If this change applies to everything, wages included, there will be no alteration in the number of transactions. Then the $f(x) d x$ transactions involving payments between $x$ and $x+d x$ will now involve payments between $k x$ and $k x+k d x$. Whence

or

$$
\begin{aligned}
& f(x) d x=\phi(k x) k d x, \\
& \phi(x) d x=f\left(\frac{x}{k}\right) \frac{d x}{k},
\end{aligned}
$$

and

$$
\phi(x)=\frac{1}{k} f\left(\frac{x}{k}\right) \text {. }
$$

Substituting this value in (B), we get as the amount of small money used

$$
2 \int_{0}^{1} x(1-x)\left[f\left(\frac{x}{k}\right)+f\left(\frac{x+1}{k}\right)+f\left(\frac{x+2}{k}\right)+\ldots\right] \frac{d x}{k} .
$$

The difference between (A) and (C) will be the alteration in the amount of small money used.

For purposes of illustration let us suppose $f(x)=C$, a constant, when $x<N$, and $f(x)=0$ when $x>N$. That is, there are no transactions involving a greater payment than $N$, and for smaller payments than $N$ those at any one figure are as numerous as those at any other. We shall suppose also that $N$ and $k N$ are integers. Then

$$
f(x)+f(x+1)+\ldots(N \text { terms })=C N,
$$

and (A) gives

$$
2 C N \int_{0}^{1} x(1-x) d x=\frac{1}{3} C N .
$$

$C N$ is the total number of transactions, and we see that each involves on the average the payment of one-third of a pound in small money. 
The series in (C) has the value

$$
f\left(\frac{x}{k}\right)+f\left(\frac{x+1}{k}\right)+\ldots(k N \text { terms })=k C N .
$$

Substituting in (C) we get

$$
2 k C N \int_{0}^{1} x(1-x) \frac{d x}{k}=\frac{1}{3} C N .
$$

There has been no change in the amount of small money required.

We can show similarly that if $f(x)$ is constant in the intervals $O N_{1}, N_{1} N_{2}, \ldots, N_{n-1} N_{n}$, where $N_{1}, N_{2}, \ldots, N_{n}$, and also $k N_{1}, k N_{2}, \ldots, k N_{n}$ are integers, there is no change in the amount of small money used due to a uniform change of prices.

Although we do not know with any degree of accuracy the form of the curve $y=f(x)$, it is obvious that it is very different from the form just assumed. The number of transactions involving the payment of sums of less than a shilling, for example, is much greater than the number involving sums between $17 \mathrm{~s}$. and $18 \mathrm{~s}$. The function $f(x)$ is undoubtedly very large in the neighbourhood of the origin, and decreases rapidly with the increase of $x$.

As a further example, we shall take

$$
f(x)=C e^{-h x},
$$

where $h$ is positive, a function which behaves in the manner just indicated, and which, with suitable values of the constants, probably approximates in some rough fashion to the reality.

The series in (A) has the value

$$
C e^{-h x}\left(1+e^{-h}+e^{-2 h}+\ldots\right)=\frac{C e^{-h x}}{1-e^{-h}},
$$

and (A) becomes

$$
\frac{2 C}{1-e^{-h}} \int_{0}^{1} x(1-x) e^{-\Lambda x} d x=\frac{2 C\left[(h+2) e^{-h}+h-2\right]}{h^{3}\left(1-e^{-h}\right)} .
$$

The series in $(C)$ is

$$
C e^{-h x / k}\left(1+e^{-h / k}+e^{-2 n / k}+\ldots\right)=\frac{C e^{-h x / k}}{1-e^{-h / k}},
$$

and $(\mathrm{C})$ becomes

$$
\frac{2 C}{k\left(1-e^{-h / k}\right)} \int_{2}^{1} x(1-x) e^{-\Lambda x / k} d x=\frac{2 C k\left[(h+2 k) e^{-h / k}+h-2 k\right]}{h^{3}\left(1-e^{-h / k}\right)} \text {. }
$$


The ratio of the quantity of small money required after the change of prices to that previously required is

$$
\frac{k\left[(h+2 k) e^{-h / k}+h-2 k\right]\left[1-e^{-h}\right]}{\left[(h+2) e^{-h}+h-2\right]\left[1-e^{-h / k}\right]} .
$$

The limit of (3) when $h$ approaches infinity is $k$. When $h$ is large the frequency curve falls rapidly to the right of the origin : hence, when the transactions involving small sums are sufficiently predominating, the amount of small money used varies with prices.

When $h$ approaches 0 , both numerator and denominator of (3) approach 0 , and we easily find, on evaluating the indeterminate form, that (3) has the value 1 . Hence, when $h$ is small, the change in the amount of small money used is slight. The frequency curve descends very slowly, and the payments involved are more uniformly distributed.

Taking $h=5$, which supposes that payments of a few pence are about 150 times as frequent as payments in the neighbourhood of $£ 1\left(e^{5}=148 \cdot 4\right)$, and supposing that prices double $(k=2)$, we get for (3) the value 1.235. The amount of small money used is increased 23.5 per cent.

In the preceding discussion one consideration has been disregarded. We assumed that if a purchase is made at the price $x$, and if the purchaser has more than $x$ in small money, precisely $x$ will be paid. But this is obviously not always the case. A person with three silver shillings cannot make a payment of seven pence. The payments of less than a shilling depend upon the payer's supply of pence in the same way as payments of less than a pound depend upon his supply of money of smaller value than a pound. The total sum required for pence payments can be evaluated from the frequency curve by taking one shilling as the unit. We have merely to put $f\left(\frac{x}{20}\right) f\left(\frac{x+1}{20}\right)$, etc., for $f(x), f(x+1), \ldots$, in formula (A).

Owing to the shorter unit used, the effect of a change of prices will be much less marked. If $k=2$, for example, the error in replacing the frequency curve by horizontal lines of unit length is quite small, and there is an approximation to the curve mentioned in the second part of the first example. 
In the second example above, taking $h=5 / 20=1 / 4, k=2$, we find for (3) the value 1.0016. Although the total amount of small money used is increased 23.5 per cent., the amount of small money used of value less than a shilling is increased by only about one-sixth of one per cent. Thus the chief effect of an increase in prices on small money is the greater use of the larger denominations of small coins.

The assumption concerning the distribution of small money:We shall now bring forward certain considerations in justification of the assumption made at the beginning of the paper that the sums of small money held by all purchasers are uniformly distributed over the interval $0-1$.

In the first place, it is easily seen that this distribution is a stable one. To take a simple example, suppose that there are $20 n$ purchasers divided into 20 groups of $n$ each, having

$$
0,1,2,3,4,5, \ldots, 18,19
$$

shillings, and that each makes a purchase of 3 shillings. After making the purchases, the groups have respectively

$$
17,18,19,0,1,2, \ldots, 15,16
$$

shillings. The distribution is uniform as before. Likewise, the purchases at any other price do not disturb the uniform distribution of small money, assuming that the number of purchases is large and the purchasers are selected at random, so that the distribution of small money among them is sensibly that of the whole.

On the other hand, suppose that the distribution is not uniform. Suppose that in the above example a larger number have 10s. than any other amount. After the purchases at 3s. the distribution will show a maximum of the same magnitude as before, but at $7 \mathrm{~s}$. Purchases at $8 \mathrm{~s} ., 18 \mathrm{~s}$, , etc., leave distributions with maxima at 2 s., 12 s., etc. ; and it is fairly obvious that on recombining all these, the maxima, appearing at different values, tend to destroy one another and to produce a more uniform distribution.

To treat the matter in its generality, let the original distribution of small money be represented by the frequency curve $y=s(x), \quad 0=x<1$. We shall suppose $s(x)$ to contain such a constant factor that $\int_{0}^{1} 8(x) d x=1$; so that of a large number, $N$, 
of persons selected at random, the number having a supply of small money lying between $x$ and $x+d x$ is $N s(x) d x$.

Now let a large number of purchases represented by the frequency curve $y=g(x)$ be made. Putting

$$
g(x)+g(x+1)+\ldots=G(x),
$$

$x<1$, the total number of these purchases for which small money between $x$ and $x+d x$ is to be paid is $G(x) d x$.

To avoid breaking integrals into two parts, let $s(x)$, which is defined only in the interval $0-1$, be defined also in the interval $1-2$ by the relation $s(x+1)=s(x)$. Of the $G(t) d t$ purchases involving small-money payments between $t$ and $t+d t$, $s(x+t) G(t) d t d x$ are made by persons having between $x+t$ and $x+t+d x$ in small money (or between $x+t-1$ and $x+t-1+d x$ if $x+t>1$ ). After the purchases these all bave between $x$ and $x+d x$ in small money (considering $d t$ to be insignificant in comparison with $d x$ ). The total number having between $x$ and $x+d x$ after all the purchases is $d x \int_{0}^{1} s(x+t) G(t) d t$. The new frequency distribution for the total number, $\int_{0}^{1} G(t) d t$, making the purchases under consideration is

$$
y=\frac{\int_{0}^{1} s(x+t) G(t) d t}{\int_{0}^{1} G(t) d t} .
$$

If $M$ and $m$ are the maximum and minimum values of $s(x)$ we have, since $G(x)$ is nowhere negative,

$$
M \int_{0}^{1} G(t) d t>\int_{0}^{1} s(x+t) G(t) d t>m \int_{0}^{1} G(t) d t .
$$

Hence in $(a)$, whatever the value of $x$ in the interval,

$$
M>y>m \text {. }
$$

Therefore the maximum of the new distribution is less than that of the old and the minimum is greater, and there is thus a more uniform distribution.

If $G(x)$ is constant we have

$$
y=\int_{0}^{1} s(x+t) d t=1
$$


and the distribution is uniform. If we represent the minimum of $G(x)$ in the interval $0-1$ by $G$ we can write $G(x)=G+G_{1}(x)$ where $G_{1}(x)=0$ in the interval. Of the total purchasers considered $G$ are reduced at once to the uniform distribution, while the remainder, $\int_{0}^{1} G_{1}(t) d t$, have a distribution varying less from the uniform than was originally the case. In a large number of purchases there will usually be some involving any sum of small money between 0 and 1 , so that in general $G$ is not sensibly zero.

When the purchasers which we have been considering are recombined with the totality of purchasers of the nation, the distribution of small money among the whole is nearer uniform. Letting $N$ represent the total number of purchasers and neglecting the influence of the $\int_{0}^{1} G_{1}(t) d t$ purchasers, the new distribution is

$$
y=\frac{(N-G) s(x)+G}{N},
$$

and the difference between the maximum and minimum values of this function is

$$
\frac{N-G}{N}(\boldsymbol{M}-m)
$$

Actually, owing to the purchasers neglected, the difference is less than this.

If a large number of successive groups of purchases be considered, $G$ having the values $G_{1}, G_{2}, \ldots, G_{n}$, the difference between the maximum and minimum values of the resulting function representing the distribution of small money is less than

$$
\frac{\left(N-G_{1}\right)\left(N-G_{2}\right) \ldots\left(N-G_{n}\right)}{N^{n}}(M-m) .
$$

This difference approaches zero as $n$ increases. From this we see that whatever may be the original distribution of small money, it becomes and remains sensibly uniform after a large number of purchases have been made. 